\title{
Disrupción tecnológica y democracia en el siglo XXI
}

\section{Technological disruption and democracy in 21 st century}

\section{Felipe Arocena}

felipe.arocena@cienciassociales.edu.uy Universidad de la República, Uruguay

Sebastián Sansone

sebastian.sansone@fder.edu.uy

Universidad de la República, Uruguay

\section{Nicolás Alvarez}

nicolasalvarezrosas@gmail.com

Centro de Altos Estudios Nacionales, Uruguay

Ministerio de Defensa, Uruguay

\section{Recepción: 01 Febrero 2021}

Aprobación: 01 Junio 2021

Publicación: 02 Agosto 2021

Cita sugerida: Arocena, F., Sansone, S. y Alvarez, N. (2021). Disrupción tecnológica y democracia en el siglo XXI. Cuestiones de Sociología, 25, e125. https://doi.org/10.24215/23468904e125

\begin{abstract}
Resumen: En el año 2019, el sociólogo Manuel Castells afirmó categóricamente que la democracia liberal agotó su recorrido histórico y, citando unos versos de Octavio Paz, expresó: "No lo que pudo ser:/es lo que fue /Y lo que fue está muerto". En este trabajo reflexionaremos sobre este diagnóstico en base a cinco preguntas interrelacionadas. ¿En qué medida la democracia liberal está siendo afectada por la actual aceleración tecnológica informacional? ¿Existe realmente una crisis democrática? ¿Qué lecciones pueden aprenderse a partir del suceso de Cambridge Analytica? ¿Será posible manipular los sentimientos: hackear humanos? ¿Cuáles son los caminos disponibles a futuro?
\end{abstract}

Palabras clave: Aceleración tecnológica, Democracia, Futuro.

Abstract: In 2019, the sociologist Manuel Castells categorically stated that liberal democracy exhausted its historical journey and, citing some verses by Octavio Paz, expressed: "Not what could be: / it is what it was / And what was is dead ". In this work we will reflect on this diagnosis based on five interrelated questions. To what extent is liberal democracy being affected by the current informational technological acceleration? Is there really a democratic crisis? What lessons can be learned from the Cambridge Analytica event? Will it be possible to manipulate feelings: hack humans? What are the paths available in the future?

Keywords: Technological acceleration, Democracy, Future.

\section{Introducción}

Al menos hasta el año 2015, internet y las redes sociales gozaron de una enorme popularidad debido a sus cualidades democratizantes. No cabía cuestionarse, o al parecer no existía suficiente evidencia que permitiese cuestionarse, sobre un posible impacto negativo (Beaumont, 2011). De hecho, durante la llamada primavera árabe (2010-2013), los medios de comunicación occidentales -y en menor medida las elites políticas y los círculos académicos (ver por ej. Manuel Castells, 2012) - se apresuraron a resaltar las virtudes de las 
revoluciones de Facebook, YouTube y Twitter. Los jóvenes "conectados por internet" pusieron fin al "despotismo y la corrupción", destacaba El País de Madrid. (Valenzuela, 2011). Las redes, aseguraba el New York Times, ofrecieron "un camino para que los descontentos se organizaran y movilizaran". Con mayor efectividad que los partidos políticos, continuaba, "la fuerza y agilidad de las redes claramente atraparon a las autoridades" (Preston, 2011). La premisa era atractiva por su sencillez: las redes dan voz a ciudadanos que se sienten poco representados por el establishment $\mathrm{u}$ oprimidos por líderes tiránicos.

El relato optimista en el que internet y las redes sociales eran un medio potente para la difusión de la democracia liberal perdió fuerza tiempo después. La historia se repetía: el homo twitter resultaba ser igual de manipulable que el homo videns. Y las redes sociales, un ágora compartida por ciudadanos comprometidos con la democracia, pero también por tiranos y extremistas. Las "alarmas democráticas" de Occidente se encendieron tras el triunfo de Donald Trump. Como se constató luego, Cambridge Analytica, coadyuvada por omisiones de seguridad de Facebook, había intentado manipular a los electores estadunidenses convirtiéndose en una pieza clave en la elección presidencial del año 2016.

Pese a las dificultades para aislar este efecto empíricamente y lograr establecer el peso de esta variable sobre el triunfo de Donald Trump, cabe cuestionarse: (i) ¿En qué medida está siendo afectada la democracia liberal por la actual revolución tecnológica informacional?; (ii) ¿Existe realmente una crisis democrática, vinculada a la aceleración tecnológica?; (iii) ¿Qué lecciones pueden aprenderse a partir del suceso de Cambridge Analytica?; (iv); y (v) ¿Cuáles son los caminos disponibles a futuro? Buscando dar una respuesta aproximada a estas interrogantes, el presente trabajo reflexiona críticamente a partir de las premisas de Yuval Noah Harari $(2015,2018)$ y Manuel Castells $(2018,2019)$ sobre la crisis del relato liberal, y en particular de la democracia. Grosso modo, de acuerdo con el primer autor, si bien resulta improbable que en las décadas venideras nos enfrentemos a una rebelión de robots, es probable que la democracia se enfrente, cada vez con mayor frecuencia, a ejércitos de bots que, utilizando la información que proporcionamos en línea, busquen vendernos ya no solo productos comerciales sino también políticos e ideologías. Y, para Manuel Castells, la democracia liberal se encuentra en una crisis de legitimidad aguda porque las instituciones en las que se montó hoy no cuentan con la confianza de la población; ni los Estados, los partidos políticos, la Iglesia católica, la justicia o los medios de comunicación cuentan con la necesaria legitimidad entre los ciudadanos, horadada en parte por la corrupción política, los escándalos mediáticos y la conciencia de la enorme desigualdad económica que caracteriza a las sociedades actuales. Tanto es así que para este sociólogo -uno de los más brillantes intérpretes de la era de la información (Castells, 1996) y entusiasta defensor del poder democratizador de internet a comienzos del milenio- hoy la democracia liberal ha agotado su recorrido histórico (Castells, 2019) y es necesario refundarla en instituciones nuevas que despierten la legitimidad de los ciudadanos en la tercera década del siglo XXI.

A continuación, se presenta un breve estado del arte acerca de la actual revolución tecnológica informacional, se realiza una reflexión sobre los límites y las posibilidades de la democracia, y se ponen a consideración los desafíos a los 
que se enfrenta este régimen político ante algunos de los avances tecnológicos más significativos.

\section{Revolución tecnológica informacional: de dónde venimos, dónde nos encontramos y hacia dónde nos dirigimos}

El punto de partida de este breve estado del arte reside en subrayar una realidad perceptible: estamos asistiendo a un proceso de transformaciones de amplitud que se impone con rapidez y la aceleración tecnológica es uno de sus principales motores. No obstante, para comprender dónde nos encontramos debemos hacer el esfuerzo de rastrear de dónde venimos y proyectar algunos posibles futuros hacia dónde nos dirigimos, sabiendo que en este último caso nos equivocaremos. A efectos analíticos, es posible argumentar que el curso de la historia humana ha estado conformado por distintas revoluciones. Las revoluciones, asegura Karl Schwab, “(...) se han producido a lo largo de la historia cuando nuevas tecnologías y formas novedosas de percibir el mundo desencadenan un cambio profundo en los sistemas económicos y las estructuras sociales" (Schwab, 2016, p. 14).

La revolución cognitiva marcó el inicio de la historia humana. El desarrollo de una postura erguida, los pulgares oponibles y principalmente los cambios en el cerebro y en la organización neurológica del sapiens conllevaron un gran salto en su capacidad cognitiva. Como consecuencia, los seres humanos lograron pensar de maneras sin precedentes, comunicarse utilizando un tipo de lenguaje nuevo y acumular culturalmente información (Harari, 2016; Bostrom, 2018). Estas capacidades permitieron el desarrollo de tecnologías más eficientes para la producción, el transporte y la comunicación, dando paso, decenas de miles de años después, a la revolución agrícola. La manipulación de distintas especies de animales y plantas mejoró la producción de alimentos, al tiempo que estimuló el crecimiento de la población facilitando el surgimiento de asentamientos humanos de mayor envergadura. La producción mecánica, marcada por la invención del motor a vapor y la construcción del ferrocarril, fue el primer paso de la revolución industrial apenas iniciada en el siglo XVIII. Posteriormente, el advenimiento de la electricidad y la cadena de montaje dieron paso a la producción en masa, el segundo gran eslabón de la revolución industrial. Finalmente, el desarrollo de los ordenadores e internet en la década de los sesenta dio lugar a la llamada tercera revolución industrial (Harari, 2014; Bostrom, 2018; Schwab, 2016). Independientemente de que aceptemos la idea de que actualmente nos encontramos en los albores de la cuarta revolución industrial, como sostiene Schwab, o aún continuemos en la tercera revolución industrial, como indica otro sector de la literatura (Rifkin, 2014), estamos, como se dijo al comienzo, frente a un conjunto de sucesos perceptible. ¿Cuáles son las características de esta nueva revolución y qué efectos podrían esperarse en el futuro cercano?

De acuerdo con Schwab (2016, pp. 17-19), la actual revolución tecnológica se caracteriza, en primer lugar, por la velocidad de innovación y difusión: "todo está ocurriendo a un ritmo mucho más rápido que nunca”. A diferencia de las anteriores revoluciones, el cambio ya no es lineal sino exponencial y marcha al mismo ritmo de la Ley de Moore, según la cual el poder computacional se duplica cada dieciocho meses (Tegmark, 2018). Las posibilidades de tener millones 
de personas conectadas mediante internet ha dado lugar a una capacidad de procesamiento y almacenamiento de datos sin precedentes. Como consecuencia, cada nueva tecnología engendra, a su vez, una tecnología más nueva y poderosa. Una segunda característica reside en la amplitud y profundidad de los cambios: "muchos cambios radicales se producen simultáneamente". La interacción de diversas disciplinas y descubrimientos de distinta índole dejaron de formar parte de la ficción para convertirse en realidades perceptibles. Actualmente, la fusión de tecnologías abarca diversos campos y se pueden destacar tres megatendencias: (i) físicas: vehículos autónomos, impresión 3D, robótica avanzada, nuevos materiales inteligentes que se autorreparan o se limpian a sí mismos; (ii) digitales: internet de las cosas; y, (iii) biológicas: manipulación genética y avances en el campo de la medicina (Schwab, 2016, pp. 22-31). Finalmente, una tercera característica se relaciona con la profunda transformación generada por el impacto de estos nuevos desarrollos. No solo asistimos a la creación de nuevos modelos de negocio y a la remodelación de los sistemas de producción, consumo y transporte, sino que, en el ámbito social, se está produciendo un cambio de paradigma en la manera en que nos comunicamos, expresamos, informamos, entretenemos, e incluso en cómo se gestionan nuestros gobiernos y las diversas áreas de política.

Pese al relativo consenso en torno a las principales tendencias actuales, no existe una única narrativa sobre cómo vivir con dichos cambios y menos aún sobre qué pasará en el futuro. Autores como Bill Joy (2000), y más recientemente Kevin Kelly (2017), llaman la atención respecto a la presencia de al menos una tríada de relatos: utópicos, distópicos y protópicos. Mientras los primeros sostienen que el progreso tecnológico -inteligencia artificial, edición genética y nanotecnologías- traerá consigo la posibilidad de trascender la evolución natural de la especie humana mediante el dominio de la selección artificial creando "súper humanos", los segundos afirman que la posibilidad de una acumulación de poder sin precedentes en manos de individuos aislados o de grupos restringidos con fácil acceso a conocimiento y materiales y poder de autoreproducción de las nuevas tecnologías supone una amenaza mayor que las armas nucleares. Finalmente, en medio de estas visiones antagónicas, quienes sostienen que la tecnología nos conducirá hacia la protopía argumentan que el futuro no es ni tan dramático ni tan excitante y convocan a la aceptación de las tendencias "inevitables" que impone la revolución tecnológica.

Dada la multiplicidad de escenarios, existe una variedad de reacciones y posiciones respecto a las posturas que se podrían asumir frente a los cambios. Desde aquellos que, acompañando el primer relato, bregan por un impulso irrestricto de las innovaciones tecnológicas, pasando por quienes perciben que la prohibición del avance tecnológico es el camino necesario, y finalmente quienes prefieren adoptar una "postura vigilante" ante las innovaciones exponenciales en inteligencia artificial, ingeniería genética y robótica, que excluya ambos extremos. Como se verá en los próximos apartados, esta variedad de relatos, desafíos y posibles caminos a recorrer, también se encuentran presentes en las discusiones relativas a la democracia. 


\section{La frontera entre lo ideal y lo real: el nudo gordiano de la democracia}

La democracia es uno de los asuntos que ha despertado mayor interés entre los estudiosos de las ciencias sociales y las humanidades. Sin la necesidad de un análisis exhaustivo, es posible identificar múltiples maneras de definir y redefinir dicho concepto. Tal es la proliferación que, en su clásico trabajo sobre la Democracia con adjetivos, Collier y Levitsky (1996) lograron constatar 550 subtipos de democracia. Lo cierto es que, luego del proceso que Huntington (1991) denominó "tercera ola de la democratización" y una vez finalizadas las diversas transiciones, la mayor parte de los países que acompañaron esa ola -muchos latinoamericanos- lograron mantener con lógicas variaciones dicho régimen político. No obstante, si miramos cualquier índice de democracia, se verá nítidamente que este ordenamiento político está casi totalmente restringido a las regiones del mundo que ubicamos en occidente, incluida América Latina ("el otro occidente") y Oceanía. En el resto del mundo la democracia es algo excepcional e infrecuente y algunos procesos de democratización más recientes que prometían, como la primavera árabe, terminaron en fracasos desestimulantes.

En la medida en que la democracia, en tanto unidad de análisis, se incrementaba, los investigadores comenzaron a realizar nuevas preguntas y utilizar nuevas variables que permitiesen complejizar y observar los distintos atributos de este "nuevo fenómeno". Un claro ejemplo de ello fue el auge de los estudios sobre calidad de la democracia (Diamond y Morlino, 2005) y déficit democrático (Beetham, 2009). No obstante, pese al incremento de estándares y herramientas de medición, el consenso en torno a la conceptualización de la democracia está muy lejos de lograrse (Diamond, 2002). Quizá, una de las claves se encuentra, como sugirió recientemente Rosanvallon (2016, p. 29), en que:

\section{Históricamente, la democracia se ha manifestado siempre como una promesa y un problema a la vez. Promesa de un régimen acorde con las necesidades de la sociedad, fundada sobre la realización de un doble imperativo de igualdad y autonomía. Problema de una realidad que a menudo está muy lejos de haber satisfecho estos nobles ideales.}

Tratándose de una construcción humana, cargada de expectativas y desencantos, precisar los alcances reales de la democracia no es tarea sencilla. Como observó Guillermo O’ Donnell, incluso si lográramos “(...) establecer un punto límite que separe todas las democracias de todas las no democracias, la ubicación de ese punto depende de las preguntas que hagamos, y por lo tanto siempre será arbitraria" (2002, p. 70).

Algunos de los eruditos más destacados de la ciencia política han dado batalla en este terreno. Norberto Bobbio (1984) llamó la atención acerca de las "falsas promesas" de la democracia, resaltando incapacidades estructurales en asuntos de participación, representación, poder y transparencia. Giovanni Sartori (1987), por su parte, realizó una distinción entre las democracias reales -tal y como existen en la realidad-y las democracias ideales -como nos gustaría que fuesen-. De forma aún más radical, Robert Dahl (1971) argumentó que la democracia no es posible, acuñando el término poliarquía.

Más recientemente, en Qué esperar de la democracia. Limites y posibilidades del autogobierno, Adam Przeworski (2010) intentó liberar a las democracias 
reales de las falsas expectativas del ideal del autogobierno. De acuerdo con el autor, la democracia representativa no ha logrado resolver, y probablemente no resolverá, cuatro desafíos que, aún en la actualidad, continúan provocando una insatisfacción muy extendida. Przeworski comienza su análisis explorando el controvertido terreno de la igualdad. Según él, una de las críticas más incisivas que recibe la democracia se funda en su incapacidad para generar igualdad socioeconómica y, como resultado de ello, forjar una situación social donde convive la igualdad política con la desigualdad social y económica. De acuerdo con el autor, en las sociedades modernas, donde la tierra ya no es la fuente de ingresos más importante, resulta difícil igualar los activos productivos. Incluso si se lograra igualar la capacidad de tener ingresos en una economía de mercado, la desigualdad resurgiría. No podemos esperar que la democracia haga lo que quizás ningún sistema de instituciones políticas puede hacer, asegura Przeworski (2010, p. 50). Otro asunto que continúa atormentando a las democracias modernas es la nostalgia de la participación efectiva. Según explica este autor, aunque en las democracias representativas los votantes tienen opciones reales, no elegirán nunca entre todas las posibilidades concebibles ya que solo es posible elegir entre las opciones propuestas. Además, a pesar de la diversidad de opciones, nadie puede en forma individual hacer que una alternativa propuesta sea la elegida. En definitiva, el segundo límite de la democracia reside en su imposibilidad de hacer sentir a la gente que su participación política es efectiva. No obstante, según Przeworski “(...) el autogobierno colectivo se logra no cuando cada votante tiene influencia en el resultado final, sino cuando la elección colectiva es resultado de la suma de las voluntades individuales" (2010, p. 167). Un tercer límite de la democracia reside en la imposibilidad de ofrecer una agentividad perfecta, es decir, asegurar que los gobiernos hacen lo que se supone que deben hacer: representar. Dado que en las democracias modernas los electores delegan sus intereses en representantes, explica Przeworski (p. 231), se espera que estos últimos representen, efectivamente, dichos intereses. Pero, en el entendido de que los representantes también poseen intereses, los costos de agentividad son inevitables. Sin embargo, la democracia es el único sistema en el que los mecanismos de accountability funcionan. Finalmente, otro desafío de la democracia reside en la dificultad de equilibrar orden con no interferencia. Según el autor, maximizar la libertad interfiriendo lo menos posible en la vida privada y garantizar, al mismo tiempo, lo máximo posible de seguridad, “(...) no es fácil de resolver y nunca podrá ser resuelto de una vez por todas” (p. 245). En la medida en que cualquier orden legal es una forma de opresión, algunas personas tendrán que vivir durante algún tiempo bajo leyes que no son de su agrado.

De este breve recorrido se pueden extraer tres conclusiones. En primer lugar, la democracia no es atemporal. Lo que resultó en Roma y Atenas (autogobierno del pueblo) no fue posible en las modernas y voluminosas sociedades industriales (democracia representativa). En segundo lugar, la democracia no es perfecta. Como vimos tiene al menos cuatro grandes límites. Finalmente, producto de estas particularidades, existe una brecha irreconciliable entre las expectativas -lo que esperamos de la democracia- y la realidad -lo que realmente sucede-. No obstante, asegura Przeworski, reconocer los límites sirve para dirigir los esfuerzos hacia ellos y, también, para mostrar las direcciones de reformas factibles (p. 53). 
Con esto, cabe cuestionarse: (i) ¿podrá la democracia gestionar los cambios que impone la disrupción tecnológica?; (ii) ¿incrementará la disrupción tecnológica los límites de la democracia?; (iii) epresenciaremos la muerte de la democracia como forma de organización política? De eso trata el siguiente apartado.

\section{La tesis de Harari: disrupción tecnológica y democracia en el siglo XXI}

Harari señala que, en su forma actual, la democracia no sobrevivirá a la fusión de la biotecnología y la infotecnología. O bien se reinventa a sí misma con éxito y de una forma radicalmente nueva, o bien los humanos acabarán viviendo en dictaduras digitales" (Harari, 2018, p. 89). Esta interpretación es diáfanamente coincidente con la de Manuel Castells que enunciamos al comienzo: o la democracia liberal se refunda o los riesgos de nuevos autoritarismos serán cada vez más frecuentes.

Aunque válidamente cuestionables, las obras de Harari (2014, 2015 y 2018) constituyen una referencia significativa para quien esté interesado en comprender y analizar críticamente el impacto de la actual revolución tecnológica informacional sobre el orden político construido por Occidente en las últimas décadas. La tecnología continúa desarrollándose en forma exponencial, mientras la democracia se muestra, a priori, bastante inmóvil y con escasa capacidad de respuesta.

En este contexto, el trabajo de Harari invita a una reflexión conjunta sobre la historia, el presente y el futuro de la humanidad. Nuestra especie, argumenta el autor en Homo Sapiens (2014), ha logrado dominar el planeta debido a su capacidad de construir relatos y cooperar en base a estos de forma flexible y en gran número. Cualquier cooperación humana a gran escala, asegura Harari, se establece en base a mitos comunes que solo existen en la imaginación colectiva: desde los dioses en la antigüedad, hasta el moderno ideal de democracia. Desde hace al menos tres siglos, asegura Harari en Homo Deus (2015), nuestras sociedades occidentales se organizan bajo un relato en el que la experiencia humana es el origen supremo de la autoridad: el humanismo. Con el tiempo, la evolución de este relato se escindió en tres ramas principales: el humanismo liberal -democracia, derechos humanos y capitalismo de libre mercado- el humanismo socialista -comunismo- y el humanismo evolutivo -fascismo-. La victoria de los aliados durante la Segunda Guerra Mundial puso fin al fascismo y con este al humanismo evolutivo, por su parte, la victoria de Occidente durante la Guerra Fría hizo lo suyo con el humanismo socialista. Desde entonces, la democracia, los derechos humanos y el capitalismo de libre mercado parecían destinados a perdurar de forma indeterminada, tal y como lo expresó Francis Fukuyama (1989) en su célebre y controvertido "fin de la historia".

No obstante, reflexiona Harari, la historia dio un giro inesperado, y ahora, tras el hundimiento del fascismo y el comunismo, el liberalismo se halla en apuros (2018, p. 14).Según el autor, el relato liberal considera el libre albedrío individual como el valor más importante. Mientras en asuntos económicos "el cliente tiene la razón”, en política "el votante sabe lo que quiere”. Desde esta concepción:

la democracia da por sentado que los sentimientos humanos reflejan un "libre albedrío" misterioso y profundo, que este "libre albedrío" es el origen último de la 
autoridad, y que mientras algunas personas son más inteligentes que otras, todos los humanos son igualmente libres. Como Einstein y Dawkins, una sirvienta analfabeta también tiene libre albedrío, de modo que el día de las elecciones sus sentimientos (representados por su voto) cuentan tanto como los de cualquier otra persona (Harari, 2018, pp. 66-7).

Este supuesto sería actualmente el talón de Aquiles de la democracia liberal. "Una vez que alguien (...) disponga de la capacidad tecnológica de acceder al corazón humano y manipularlo, la política democrática se transformará en un espectáculo de títeres emocional”, asegura Harari (2018, p. 67). El hecho de que los electores puedan estar sujetos a manipulación no es una preocupación nueva. En un artículo escrito hace más de medio siglo, Joseph Schumpeter, uno de los precursores de la teoría de la democracia, sostenía que la información y los argumentos que se le presentan a los electores están siempre al servicio de una intención política. $\mathrm{Al}$ respecto, aseguraba:

el ciudadano normal desciende a un nivel inferior de prestación mental tan pronto como penetra en el campo de la política (...) argumenta y analiza de un modo que él mismo calificaría de infantil si estuviese dentro de la esfera de sus intereses efectivos (1942, p. 235).

Igual de escéptico fue Sartori (1998) al escribir Homo videns. Según este, con el desarrollo de la $T V$ el homo sapiens entró en crisis; una crisis de pérdida de conocimiento y de capacidad de saber. La exposición permanente al bombardeo de imágenes trajo aparejada una pérdida en la capacidad de abstracción y de raciocinio, produciendo un ser apático y manipulable. Pese a que el autor concentró sus esfuerzos en el análisis del impacto de la televisión, reflexionó sobre el futuro de una idea que, al momento de su análisis, era incipiente: internet. En teoría, aseguraba, internet debería estimular el crecimiento cultural. Pero en la práctica puede suceder lo contrario, desde el momento en que el homo videns ya está formado cuando se enfrenta a la red (Sartori 1998, p. 55). Al respecto, sentencia:

sea como fuere, para los comunes mortales la navegación cibernética es sólo una especie de videojuego. Y si toman esta navegación demasiado en serio, los cibernautas «comunes» corren el riesgo de perder el sentido de la realidad, es decir, los límites entre lo verdadero y lo falso, entre lo existente y lo imaginario. Para ellos todo se convierte en trampa y manipulación y todo puede ser manipulado y falseado (1998, p. 58$){ }^{1}$

Es necesario ahora retomar algunas de las interrogantes planteadas con anterioridad: (i) ¿en qué medida (cómo y por qué) está siendo afectada la democracia liberal por la actual revolución tecnológica informacional?; (ii) ¿son internet y las redes sociales los nuevos agentes de manipulación?; y (iii) ¿será posible manipular los sentimientos, hackear humanos?

No es fácil probar empíricamente cuánto influyó Cambridge Analytica en el triunfo de Donald Trump, si realmente las campañas rusas de desinformación han sido determinantes en algún resultado político-electoral, ${ }^{2}$ o si los grupos de WhatsApp de Jair Bolsonaro en Brasil inclinaron la balanza en su favor. ${ }^{3}$ Tampoco es posible predecir con certeza si alguien dispondrá en el futuro de la capacidad tecnológica de acceder al corazón humano y manipularlo, como argumenta Harari (2018). O si, como asegura Ian Morris (2016), la 
democracia desaparecerá cuando quede al descubierto su incapacidad para resolver los problemas del futuro, dejando nuestras decisiones políticas en manos de algoritmos. Pese a las dificultades para establecer el grado de influencia del fenómeno, es decir, para probar cuánto influye, es posible destacar tres tendencias empíricas para reflexionar sobre el porqué de dicha incidencia: (a) nuestras decisiones más mundanas como, por ejemplo, buscar información y debatir sobre asuntos políticos, dejan al descubierto una marcada dependencia a distintos tipos de IA, como los motores de búsqueda y las redes sociales; (b) producto de lo anterior, transferimos importantes volúmenes de información personal a terceros; y (c) dicha información, además de ser valiosa comercial y políticamente, se concentra en una elite minúscula con la capacidad necesaria para su procesamiento. ¿Pero cómo inciden estas tendencias en la democracia? De acuerdo con la tesis bajo la que se sustenta este trabajo existen, en principio, dos riesgos: (a) pérdida de libertad frente a los algoritmos: escuch a al algoritmo y (b) pérdida de igualdad frente a los datos: quienes poseen los datos poseen el futuro (Harari, 2015 y 2018).

En una de sus más recientes obras, Cathy O’ Neil (2016) analizó el impacto del big data sobre la democracia. Mediante el análisis de estudios realizados por las propias compañías tecnológicas argumenta que pese a la inexistencia de pruebas que demuestren que Facebook, Google o Amazon estén utilizando sus IA para causar daño, el potencial de abuso es enorme. Generalmente, asegura la autora, las compañías están enfocadas en ganar dinero. Sin embargo, advierte, sus beneficios están estrechamente vinculados a las políticas gubernamentales, por ejemplo a las regulaciones fiscales. Al banalizar el poder de estas compañías, asegura $\mathrm{O}^{\prime}$ Neil, perdemos de vista dos asuntos relevantes. Por un lado, los algoritmos no son neutrales. Las compañías determinan, según sus propios intereses, lo que vemos y aprendemos en sus redes sociales o buscadores. Por otro lado, su potencial no proviene únicamente de su alcance sino también de su capacidad para influir en sus propios clientes y utilizar a sus propios clientes para influir en sus amigos. A modo de ejemplo, la autora cita dos experimentos sociales realizados recientemente. El primero fue llevado adelante por Facebook en el año $2012^{4}$ e involucró a 680.000 usuarios con el objetivo de determinar si las actualizaciones en sus noticias podían afectar su estado de ánimo. Utilizando un software lingüístico, Facebook clasificó las actualizaciones positivas y negativas para posteriormente exponer a los usuarios a ellas. Al evaluar el comportamiento posterior, hallaron evidencia que probaba su hipótesis sobre los cambios en los estados de ánimo: quienes fueron expuestos a actualizaciones negativas produjeron más contenido negativo y viceversa. Su conclusión: "Los estados emocionales pueden transferirse..., lo que lleva a las personas a experimentar las mismas emociones sin su conciencia" (O'Neil, 2016, pp. 156-157). De un modo similar, otra investigación realizada por dos académicos estadounidenses logró determinar el potencial de Google para incidir sobre el voto individual. Los investigadores solicitaron a votantes indecisos que utilizaran un motor de búsqueda para informarse sobre las próximas elecciones. Los motores que utilizaban estaban programados para sesgar los resultados de búsqueda favoreciendo a una parte sobre otra. Esos resultados, afirmaron los investigadores, cambiaron las preferencias de voto en un $20 \%$ (O’Neil, 2016, p. 157). 
El asunto de fondo reside, como argumenta Harari, en que tendemos a pensar que las IA se revelarán en nuestra contra cuando el problema real es justo el contrario: debemos temerles porque probablemente obedecerán siempre a sus amos y nunca se revelarán. No hay nada de malo en la obediencia ciega, desde luego, mientras los robots sirvan a amos benignos (Harari, 2018, p. 84). A estos desafíos en el terreno de la libertad y la igualdad, se suman otros referentes a la representación y la participación. Tras el suceso de Cambridge Analytica, internet y las redes sociales se hallan en el ojo de la tormenta. La presencia de ejércitos de bots, trolls y fakes al servicio de líderes de dudosa reputación democrática, los casos de injerencia extranjera y la generación de las llamadas "burbujas de la información" -responsables del incremento de la polarización y la intolerancia-, son algunos de los efectos visibles del mal uso de la red.

La actual crisis de representación que sufre la democracia no ha afectado únicamente al sistema político. Todo aquel interlocutor percibido como aliado del establishment ha sido objeto de desacreditación, tal es el caso de los medios de comunicación tradicionales. Una de las tesis, sostenidas por el relato optimista, aseguraba que la "revolución de internet" permitiría que cada usuario fuese una fuente inagotable de noticias. En teoría, el incremento de la participación y la difusión de información a manos de los propios ciudadanos/electores, sería un remedio exitoso para aquellos que no se sintiesen representados o fuesen oprimidos por gobiernos antidemocráticos y sus socios de los medios de comunicación. No obstante, en la práctica, la tesis padece de al menos tres puntos ciegos. Por un lado, el hecho de que cada ciudadano/elector pudiese generar noticias dejó al descubierto la infinidad de usuarios dispuestos a crear un contenido nocivo con fines de desinformación. Por otro lado, el hecho de generar contenidos propios no garantiza a los usuarios su control. Como se vio anteriormente, las compañías comenzaron a desarrollar algoritmos que filtran la información en nuestro nombre. Finalmente, y en relación a lo anterior, la red no garantiza la exclusión de intereses perversos ya sean empresariales o políticos.

En definitiva, como se intentó demostrar, la democracia se ha visto afectada de diversas maneras. Hasta el momento, algunas de las compañías involucradas han realizado extensos mea culpa, prometiendo implementar medidas para mitigar los efectos negativos. ${ }^{5}$ No obstante, estas continúan teniendo el control de la información y un potencial de abuso sin precedentes. Pensar que por sí solas solucionarán el problema es un peligroso llamado a la complacencia.

\section{Reflexiones finales y conclusión}

El filósofo francés Éric Sadin escribió pocos años atrás un libro titulado La silicolonizacin del mundo. Habla allí de un espíritu liberal de aventuras. Ese mismo espíritu aventurero estadounidense del siglo XIX que posibilitó la expansión hacia la costa oeste y que habilitó que se extrajera oro de forma indiscriminada, hoy se repetiría en Silicon Valley y de allí al mundo todo a través de la aceleración tecnológica y la economía del dato. Esta aventura tecnológica es impulsada por un grupo muy particular de jóvenes graduados universitarios, con jornadas laborales extensas y flexibles y un nivel de confort impensado por las generaciones de sus padres. Motivados por la promesa de mejorar su calidad de vida, de ascenso y reconocimiento social rápido, estos jóvenes han 
logrado modificar la lógica de funcionamiento del capitalismo actual, impulsando la recolección masiva de datos. Como si de nostalgia del pasado se tratara, a esto se le ha puesto el nombre de data mining, o minería de datos, pero hoy con información personal, suya, mía, de todos. Y algo más, el lugar donde todo esto transcurrió en su comienzo fue apodado como el valle del silicio, el mineral esencial para producir los microprocesadores y otros componentes de la computación (Sadin, 2018, p. 27).

Este espíritu ha trascendido las fronteras norteamericanas y se ha instalado como un nuevo paradigma de producción económica, organización social y dominación política. Cada vez es más común la sustitución de los obreros trabajando en fábricas por robots operados por computadoras con inteligencia artificial, mucho más efectivos y menos "problemáticos". Esto se puede apreciar visualmente en las nuevas ciudades tecnológicas inteligentes, o tecnópolis, como la carretera 128 de Boston, Akademgorodok en Rusia, o Tsukuba en Japón. ${ }^{6}$ Además, la política ha entrado en una fase algorítmica en la que buena parte de los votantes son seducidos por mensajes diseñados a su medida por sistemas tecnológicos que los conocen más que ellos mismos. Detrás de estas innovaciones subyace una visión del mundo que se puede sintetizar como el tecnoliberalismo.

El tecnoliberalismo es la filosofía predominante de Silicon Valley, que asimila una vertiente actualizada del liberalismo económico de los clásicos junto con una nueva fe en la potencialidad liberadora de la tecnología. La ontología tecnolibertaria consiste en descalificar la acción humana en beneficio de un ser "computacional superior", escribe (Sadin, 2018, p. 126). El objetivo final de esta filosofía sería despojarse de las ataduras de lo político y avanzar en la ruta para la emancipación humana del Estado, de la clase política y de las normas judiciales. Para eso se apela a un sistema digital inteligente superior, capaz de constituirse en una especie de conciencia colectiva global con la mayor autonomía y capacidad de seleccionar lo mejor para la humanidad dentro de un conjunto de escenarios posibles. ${ }^{7}$ Patri Friedman (nieto de Milton Friedman y antiguo ingeniero de Google) lo confiesa cabalmente: hay tantas cosas importantes y que nos entusiasman que podríamos hacer, pero no podemos porque serían ilegales (Sadin, 2018, p. 127).

En sus comienzos, hacia fines del siglo XX, el potencial democratizador de internet era uno de los principales atractivos de la red global. Se destacaba una y otra vez que cualquiera podría acceder a información ilimitada de manera casi gratuita, que el conocimiento es poder y que ese poder se repartiría de forma horizontal habilitando el empoderamiento de los ciudadanos. Así lo veían desde Manuel Castells, uno de los pioneros en analizar a fondo la era de la información, a Steve Jobs, el fundador de Apple; desde Bill Gates, el multibillonario dueño de Microsoft, a Jeremy Rifkin, exégeta de la tercera revolución industrial digital. Precisamente este último hablaba entonces del poder lateral: un poder colaborativo liberado por la unión de la tecnología de internet y de las energías renovables [que] reestructura radicalmente las relaciones humanas haciendo que, de verticales (desde arriba), se conviertan en horizontales (de lado a lado) (Rifkin, 2014, p. 19). Castells también describía con lujo de detalles esta horizontalidad del poder; el nuevo prototipo de la organización social era la red, sin un único centro sino con múltiples nodos. Así empezaban a vincularse las comunidades científicas, los grupos de consumidores, 
los activistas sociales y los votantes, a través de la interacción en red. Estamos hablando del auge de los foros, los blogs o comienzo del intercambio persona a persona (p2p) que llevó a populares juicios por derechos de autor, como el caso de Metallica vs. Napster. Aunque esta perspectiva pueda sonar ingenua en la tercera década del siglo XXI, todavía hay acontecimientos recurrentes que le dan su lugar. Por ejemplo, las movilizaciones antiracistas en Occidente en 2020 por la muerte de los dos hombres afrodescendientes a manos de los policías en Estados Unidos o, también, hace algunos años, la llamada primavera árabe, o el movimiento Occupy Wall Street. ¿Por qué entonces este inicial potencial liberador de internet hoy suena ciertamente ingenuo?

Las sospechas y críticas sobre esa promesa de democratización chocan en el presente con la obscenidad de la concentración de poder; acumulación de poder económico, de poder tecnológico y de poder de información. Son las llamadas cuatro grandes las que dominan esta nueva etapa de inteligencia artificial: Google, Facebook, Amazon y Apple; son sus fundadores los que poseen un porcentaje escandaloso de la riqueza global; y son sus sistemas digitales los que acceden y controlan los datos personales de la mayoría de la población mundial. En los regímenes totalitarios, donde algunas de estas empresas son impedidas de funcionar, es en el propio Estado y sus aliados donde se concentra el poder, la riqueza y la información. El Estado chino se ha visto robustecido por la tecnología, y no al revés, como se especuló previamente. China ha incorporado, por ejemplo, el llamado crédito social, donde el comportamiento de buen comunista queda registrado en un puntaje que beneficiará o no a los habitantes para cada compra que haga, a la hora de iniciar gestiones, tener o no que pagar una garantía para el alquiler de un auto, e incluso para viajar libremente entre provincias:

Queridos pasajeros, aquellos que viajen sin billete, que se comporten desordenadamente o que fumen en lugares públicos serán castigados de acuerdo a las reglas y su comportamiento quedará registrado en el sistema de créditos e información individual. Para evitar cualquier registro negativo en su crédito personal siga las normas y cumpla las órdenes en el tren y la estación (audio en un tren de Shanghai de 2018). ${ }^{8}$

Aunque la polisemia de la democracia es alta, puede condensarse como un cierto régimen político que empodera ciudadanos porque tienen los derechos de elegir sus representantes y ser electos, en un contexto social de libertades, con individuos relativamente autónomos que creen saber o sentir lo que les parece mejor, con partidos políticos compitiendo por su voto. Para que una democracia funcione más o menos bien debe existir, antes que nada, como lo destacó Max Weber, la creencia en la legitimidad de la trama institucional que la garantiza. Sin esta creencia no es posible sostenerla.

Este es uno de los principales desafíos para la democracia en el contexto de silicolonización. Es que justamente la gente ha dejado de creer en las instituciones que cementaron estos regímenes políticos en el siglo pasado. Los partidos políticos no tienen credibilidad, los parlamentos no merecen la confianza de los votantes y los candidatos son vistos como personas que buscan sus propios intereses. Esto puede constatarse fácilmente en muchas de las encuestas de opinión realizadas en los últimos años en casi todas partes del mundo donde se hacen sondeos de este tipo sin censura. Las instituciones que forjaron la 
democracia como el mejor de los regímenes políticos conocidos no gozan más de la confianza de la gente. $\mathrm{O}$, dicho de otra manera, la ciudadanía ha puesto entre paréntesis la creencia en su legitimidad (Castells, 2019). Para ello ha contribuido no en poca medida la avalancha de información y desinformación que circula en las redes sociales que, al decir de Umberto Eco, "les dan espacio a legiones de idiotas".

Un segundo desafío, tan fundamental como la recuperación de la confianza en las instituciones, y emparentado con ello, refiere a la autonomía de las personas para saber qué es lo que quieren o sienten. Lo que está ocurriendo con la aceleración de las tecnologías de inteligencia artificial, y esto lo explica muy bien Yuval Noah Harari, es que están poniendo radicalmente en duda esta segunda creencia. Ya no son los ciudadanos los que mejor saben lo que desean o lo que sería mejor para ellos, sino que los algoritmos que procesan toda nuestra información personal tienen conclusiones mucho más certeras sobre lo que somos, sentimos y queremos. Mediante el análisis de nuestras huellas dejadas en los dispositivos digitales que nos acompañan todos los días las veinticuatro horas no hay engaños ni equivocaciones. La información está ahí, no podemos mentirnos más a nosotros mismos porque el espejo de lo que somos en verdad lo tiene construido un sistema de inteligencia artificial que sabe más de nosotros que nosotros mismos. ¿A quién no le pasó que luego de una búsqueda de compra en internet comenzó a recibir recomendaciones de las mejores posibilidades para su tipo familiar? Esto se repite en todos los planos de la vida, desde la política al amor, del turismo a la lectura, desde la religión a la vivienda o la salud.

Allí radica la tentación de los jóvenes tecnoliberales que nos han colonizado desde Silicon Valley, ellos y sus tecnologías creen que están a punto de construir un mundo nuevo mucho mejor. Si será mejor, no lo sabemos, pero que ya es radicalmente nuevo no hay dudas.

Y todo lo anterior hace pensar, siguiendo el pensamiento de Harari y Max Tegmark, los cuales mantuvieron una discusión interesantísima respecto a la democracia y la IA, ${ }^{9}$ que se puede decir que la democracia tiene los días contados. Tegmark observa que en los debates entre Trump y Clinton no hubo mención a la IA, ni siquiera cuando hablaron de empleo. Y Harari, aún más duro, afirma que, a diferencia de los eventos que nos precedieron, ahora y en el futuro mucho más eficientemente, habrá un sistema informático que sabrá más y mejor acerca de nosotros que nosotros mismos. Ni dios ni el emperador sabían acerca de nosotros; la IA puede relevar un perfil sobre mi persona en segundos, y se pregunta:

¿cómo funciona la democracia en un mundo donde alguien entiende al votante mejor de lo que el votante se entiende a sí mismo? Y lo mismo con el libre mercado. Quiero decir, si el cliente no tiene razón, si el algoritmo es correcto, entonces necesitamos un sistema económico completamente diferente. Esa es la gran pregunta en la que creo que deberíamos centrarnos. No tengo la respuesta, pero cualquier historia que sea relevante para el siglo XXI tendrá que responder esta pregunta (Harari, 2014).

Si bien el concepto de silicolonización del mundo puede ser seductor para explicar determinadas cosas - como la fragilidad de ciertos Estados nacionaleses importante notar que su afectación se da cuando los sistemas de gobierno son abiertos, como es el caso de la democracia. En los sistemas políticos cerrados, por el contrario, parece que la lógica es inversa: sirve para permitir la autopoiesis de ese mismo sistema cerrado, perfeccionándolo y expandiéndolo. 
La democracia se encuentra en crisis. No es novedad, pero ahora se le suma el impacto de la voracidad de las tecnologías de la información de última generación. Pese a sus límites, hasta el momento ningún proyecto político ha logrado otorgar en forma simultánea mayores posibilidades de participación, representación, libertad e igualdad. No obstante, en lo inmediato, la falta de reformas, el escepticismo y las expectativas irracionales continúan dando lugar a campañas populistas. Hechos de reciente configuración, han dejado al descubierto la otra cara del mundo digital. En momentos de escepticismo y una marcada dependencia de distintos tipos de IA en una red repleta de bots, trolls y fakes recrudecen estándares inéditos de polarización e intolerancia que se cristalizan con la llegada al poder de líderes de dudosa reputación democrática.

La confianza ciega en las "tecnologías para la liberación" y una banalización de los efectos negativos del mal uso de la red y del big data ha provocado un importante desfasaje. Como han reconocidos distintos eruditos, la democracia actual carece de metodologías que le permitan blindarse ante la combinación tóxica de estos factores. ${ }^{10}$ Solemos creer que las democracias mueren de forma espectacular, a mano de hombres armados; el problema es que la democracia puede morir en manos de líderes electos que se escudan en la red. ${ }^{11}$

Quizá, como aseguró recientemente Kofi A. Annan, la clave se encuentre en que si la tecnología no se detiene, tampoco debe hacerlo la democracia. ${ }^{12} \mathrm{Se}$ reinventa o está destinada a perecer.

\section{Referencias}

Beaumont, P. (2011): The truth about Twitter, Facebook and the uprisings in the Arab world. Recuperado https://www.theguardian.com/world/2011/feb/25/twitterfacebook-uprisings-arab-libya

Beetham, D. (2009). Problems in identifying a democratic déficit. En XXI Congreso Mundial de Ciencia Política IPSA, Santiago, 12-16 de julio.

Bobbio, N. (1984). El futuro de la democracia. México DF: Fondo de Cultura Económica.

Bostrom, N. (2018). Superinteligencia. Madrid: Teell Editorial.

Cansino, C. (2016). Viejas y nuevas tesis sobre el Homo Twitter. En Del Homo Videns al Homo Twitter. Democracia y redes sociales. México: Benemérita Universidad Autónoma de Puebla.

Castells, M. (1996). La era de la información. México: Siglo XXI.

Castells, M. (2012). Comunicación y poder. Madrid: Alianza.

Castells, M. (2018). Ruptura. La crisis de la democracia liberal. Madrid: Alianza.

Castells, M. (2019). Conferencia Crisis de la democracia liberal global. Valparaíso: Puerto de Ideas. Recuperado de https://www.youtube.com/watch?v=q9blcS9bx $\mathrm{O} 0$

Castells, M. y Hall, P. (1994). Las tecnópoolis del mundo. Madrid: Alianza.

Collier, D. y Levitsky, S. (1996). Democracy With Adjective: Conceptual Innovation in Comparative Research. Berkeley: Universidad de California.

Dahl, R. (1971). Polyarchy. Participation and Opposition. New Haven: Yale University Press. 
Diamond, L. (2002). Elections Without Democracy: Thinking About Hybrid Regimes. Journal of Democracy, National Endowment for Democracy and the Johns Hopkins University Press, vol. 13.

Diamond, L. y Morlino, L. (2005). Assessing the Quality of Democracy: Theory and Empirical Analysis. Baltimore: The Johns Hopkins University Press.

Fukuyama, F. (1989). The end on history?. En The National Interest (pp. 3-18).

Harari, Y. N.. (2014). Sapiens: De animales a dioses. Madrid: Debate.

Harari, Y. N. (2015). Homo Deus. Breve historia del mañana. Madrid: Debate.

Harari, Y. N. (2018). 21 lecciones para el siglo XXI. Madrid: Debate.

Huntington, S. (1991). The Third Wave: Democratization in the Late Twentieth Century. Norman, University of Oklahoma Press, vol. 15.

Joy, B. (2000). ¿Por qué el futuro no nos necesita?. Wired Magazine.

Kelly, K. (2017). Lo inevitable. Entender las 12 fuerzas tecnológicas que configurarán nuestro futuro. Madrid: Teell Editorial.

Levitsky, S. y Ziblatt, D. (2018). Cómo mueren las democracias. Buenos Aires: Ariel.

Morris, I. (2016). La democracia y el desafío tecnológico. En Del Homo Videns al Homo Twitter. Democracia y redes sociales. México: Benemérita Universidad Autónoma de Puebla.

O' Donnell, G. (2002). Ilusiones sobre la consolidación. Nueva Sociedad, 180-181.

O' Neil, C. (2016). Weapons of Math Destruction. New York: Crown.

Preston, J (2011): Movement Began With Outrage and a Facebook Page That Gave It an Outlet. Recuperado de: https://www.nytimes.com/2011/02/06/world/middleea st/06face.html

Przeworski, A. (2010). Qué esperar de la democracia. Limites y posibilidades del autogobierno. Buenos Aires: Siglo Veintiuno.

Rifkin, J. (2014). La Tercera Revolución Industrial. Barcelona: Paidós.

Rosanvallon, P. (2016). La democracia en la era de la desconfianza. En Del Homo Videns al Homo Twitter. Democracia y redes sociales. México: Benemérita Universidad Autónoma de Puebla.

Sadin, E. (2018). La silicolonización del mundo. Buenos Aires: Caja Negra.

Sartori, G. (1987). The Theory of Democracy Revisited. N.J: Chatham House Publishers.

Sartori, G. (1998). Homo videns. Buenos Aires: Aguilar.

Schumpeter, J. (1942) [1996]. Capitalismo, socialismo y democracia. Barcelona: Ediciones Folio.

Schwab, K. (2016). La cuarta revolución industrial. Barcelona: Debate.

Snyder, T. (2018). The road to unfreedom. New York: Penguin Random House.

Tegmark, M. (2018). Vida 3.0. ¿Qué significa ser humano en la era de la inteligencia artificial?. Barcelona: Taurus.

Valenzuela, J. (2011). Europa y la revolución democrática árabe. Recuperado de a https ://elpais.com/diario/2011/01/29/opinion/1296255611_850215.html

\section{Notas}

1 La tesis optimista desarrollada por César Cansino (2016) asegura que el homo twitter superó al homo videns destacando los beneficios de las redes sociales en política.

2 Al respecto puede verse el reciente libro de Timothy Snyder (2018). 
3 Puede consultarse en línea: https://www.nytimes.com/es/2018/10/23/brasil-whatsa pp-noticias-falsas/

4 Los resultados pueden consultarse en línea: https://www.pnas.org/content/111/24/ 8788.full

5 En una serie de columnas agrupadas bajo el título Preguntas Difíciles: ¿Qué impacto tienen las redes sociales en la democracia?, Facebook reconoció "las influencias negativas" de las redes sociales para "el bienestar democrático". Consultar en línea: https://ltam.newsroom.fb.com/news/h/preguntas-dificiles-que-impacto-tiene n-las-redes-sociales-en-la-democracia/

6 Para profundizar más sobre la creación, consolidación y expansión de las tecnópolis, ver Castells y Hall, Las tecnópoolis del mundo, 1994.

7 El film Trascendence (2014), precisamente, indaga sobre este tema. Se puede ver su argumento en https://www.imdb.com/title/tt2209764/

8 Diario El Mundo, 31 octubre 2018.

9 Disponible en: https://futureoflife.org/2019/12/31/on-consciousness-morality-effec tive-altruism-myth-with-yuval-noah-harari-max-tegmark/

10 Puede consultarse en línea: https://www.idea.int/es/news-media/news/es/el-estadode-la-democracia-\%C2\%BFestancamiento-regresi\%C3\%B3n-o-resiliencia

$11 \mathrm{Al}$ respecto puede verse el reciente libro de Levitsky y Ziblatt (2018).

12 Puede consultarse en línea: https://www.nacion.com/opinion/columnistas/como-las -redes-sociales-amenazan-la-democracia/FF6G6A2MZNEWFN5YV5LQZ3YK4E/ story/ 